\title{
Combined Inclusive Diffractive Cross Sections Measured with Forward Proton Spectrometers in $e p$ DIS at HERA
}

\section{J. Olsson (on behalf of the $\mathrm{H} 1$ and ZEUS Collaborations)}

DESY, Hamburg, Germany

E-mail: olsson@mail.desy.de

A combination of the inclusive diffractive cross section measurements made by the $\mathrm{H} 1$ and ZEUS Collaborations at HERA is presented. The analysis uses samples of diffractive deep inelastic $e p$ scattering data at a centre-of-mass energy $\sqrt{s}=318 \mathrm{GeV}$ where leading protons are detected by dedicated spectrometers. Correlations of systematic uncertainties are taken into account, resulting in an improved precision of the cross section measurement which reaches $6 \%$ for the most precise points. The combined data cover the range $2.5<Q^{2}<200 \mathrm{GeV}^{2}$ in photon virtuality, $0.00035<x_{\mathbb{P}}<0.09$ in proton fractional momentum loss, $0.09<|t|<0.55 \mathrm{GeV}^{2}$ in squared four-momentum transfer at the proton vertex and $0.0018<\beta<0.816$ in $\beta=x / x_{\mathbb{P}}$, where $x$ is the Bjorken scaling variable.

XXI International Workshop on Deep-Inelastic Scattering and Related Subjects - DIS2013, 


\section{Introduction}

The diffractive deep inelastic ep scattering (DIS) process can be schematically described as follows (figure 1): The scattered electron radiates a virtual photon, which reacts with a parton from the colour singlet object, which is emitted by the scattered proton. Usually this colour singlet object is called "pomeron", after the great Russian physicist I. Pomerantschuk. The photon pomeron interaction produces the hadronic system $X$, which, due to the pomeron being colourless, is clearly separated from the scattered proton by a "rapidity gap", also when the proton dissociates into a low mass system. The experimental signature of a large rapidity gap (LRG) can be used as selection criterium for diffractive $e p$ DIS events.

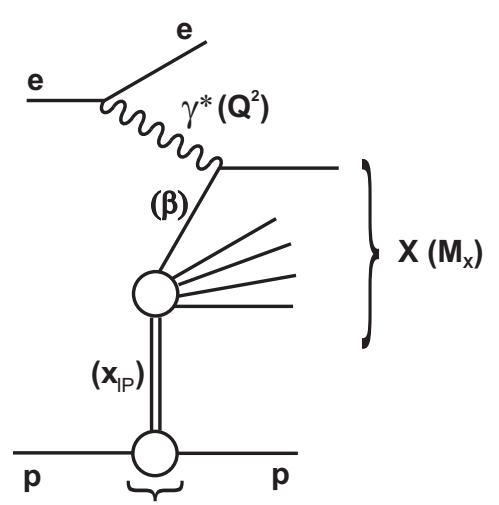

(t)

Figure 1:

Diagram of the reaction $e p \rightarrow e X p$.

That LRG events constitute a substantial fraction of the total DIS ep cross section was discovered already in the beginning of the HERA operation, and the study of diffractive $e p$ scattering has since been an important part of the HERA physics program. The diffractive reactions provide a tool for investigating the low momentum partonic structure of the proton, and thus also the partonic structure of the pomeron itself [1]. In such studies, the photon virtuality $Q^{2}$ serves as a hard scale for perturbative QCD calculations and both the H1 and ZEUS collaborations have used their high statistics LRG data samples to extract diffractive parton distribution functions (DPDFs), through NLO DGLAP fits[2, 3]. However, there is a substantial uncertainty in such analyses, since the LRG data give no direct information about that part of the cross section, where the proton dissociates into a system $Y$. If the mass $M_{Y}$ of this system is large, several GeV say, then such an event may be tagged through hadrons triggering one or several of the forward parts of the main detector. Using forward-tagged data, the uncertainty can be estimated through the use of model calculations, but what is needed is clearly a proton detector, which is triggered by the scattered proton alone. With such data, it is possible to normalise the LRG data, and quantify the fraction of proton dissociation events.

During the years of HERA operation both H1 and ZEUS operated such detectors, known as Forward (Leading) Proton Spectrometers, FPS (LPS), respectively. In this paper, a combination of the results from four different FPS and LPS data analyses is presented. For details more than can be given here, please see [4].

\section{Kinematic Variables and Cross Sections}

The diffractive process in figure 1 is described in terms of $Q^{2}$ and $\beta$, where $\beta$ is the momentum fraction of the interacting parton w.r.t. the pomeron; thus $\beta$ plays the same role as Bjorken $x\left(x_{B j}\right)$ in classical DIS. Furthermore, $M_{X}$ is the mass of the produced hadronic system $X$ and $x_{\mathbb{P}}$ is the momentum fraction of the proton, carried by the pomeron. Note that $\beta \cdot x_{\mathbb{P}}=x_{B j}$ and $\beta \simeq Q^{2} /\left(Q^{2}+M_{X}^{2}\right)$. The squared momentum transfer at the proton vertex is denoted by $t$. If the 


\begin{tabular}{|l|c|c|c|c|}
\hline Data Set Name & H1 FPS HERA II & H1 FPS HERA I & ZEUS LPS 2 & ZEUS LPS 1 \\
\hline$Q^{2}$ range $\left[\mathbf{G e V}^{2}\right]$ & $4-700$ & $2-50$ & $2.5-120$ & $2-100$ \\
\hline$x_{\mathbb{P}}$ range & $<0.1$ & $<0.1$ & $0.0002-0.1$ & $<0.1$ \\
\hline$y$ range & $0.03-0.8$ & $0.02-0.6$ & & \\
$W$ range $[\mathrm{GeV}]$ & & & $40-240$ & $25-240$ \\
\hline$\beta$ range & $0.001-1$ & $0.004-1$ & & \\
$M_{X}$ range $[\mathbf{G e V}]$ & & & $2-40$ & $>1.5$ \\
\hline$|t|$ range $\left[\mathbf{G e V}^{2}\right]$ & $0.1-0.7$ & $0.08-0.5$ & $0.09-0.55$ & $0.075-0.35$ \\
\hline Int. Lumi. $\left[\mathbf{p b}^{-1}\right]$ & 156.6 & 28.4 & 32.6 & 3.6 \\
\hline Data Taking Year & $2006-2007$ & $1999-2000$ & $1999-2000$ & 1997 \\
\hline
\end{tabular}

Table 1: H1 [6], [5] and ZEUS [2], [7] data sets used for the combination.

proton stays intact in the interaction, then $M_{Y}=M_{\text {proton }}$. Finally, $W_{\gamma p}=x_{B j} \cdot y \cdot s$ is the energy of the $\gamma p$ Centre-of-Mass system; here $y$ is the inelasticity and $s$ the total ep CM energy. Note that the variables $Q^{2}, W_{\gamma p}$ and $x_{B j}$ are reconstructed using measurements with the main detector, while $\beta$, $x_{\mathbb{P}}, t$ and $M_{X}$ are reconstructed from proton spectrometer measurements or from a combination of these with measurements in the main detector.

The diffractive inclusive cross section is usually expressed with help of the reduced diffractive cross section $\sigma_{r}^{D(4)}$ :

$$
\frac{\mathrm{d} \sigma^{e p \rightarrow e X p}}{\mathrm{~d} \beta \mathrm{d} Q^{2} \mathrm{~d} x_{P} \mathrm{~d} t}=\frac{4 \pi \alpha^{2}}{\beta Q^{4}}\left[1-y+\frac{y^{2}}{2}\right] \quad \sigma_{r}^{D(4)}\left(\beta, Q^{2}, x_{\mathbb{P}}, t\right) .
$$

After integrating over $t$, the reduced cross section $\sigma_{r}^{D(3)}\left(\beta, Q^{2}, x_{P}\right)$ is obtained. In the following, results are presented in terms of $\sigma_{r}^{D(3)}$. As is wellknown, at small and moderate values of the inelasticity $y, \sigma_{r}^{D(3)}$ is in good approximation equal to the diffractive structure function $F_{2}^{D(3)}$.

\section{The FPS/LPS Detectors and H1/ZEUS Data Sets}

Both H1 FPS and ZEUS LPS spectrometers use Roman Pot technology, and are installed in spaces inbetween magnets of the HERA beam line, in 60-90 m distance downstream of the main H1 and ZEUS detectors. While the H1 FPS detectors are based on scintillating fibres and PSPMs (Position Sensitive PhotoMultipliers), the ZEUS LPS are built with Silicon Microstrip technology. The various stations register the position of the passage of the scattered proton, and since the HERA beam line serves as a magnetic spectrometer, a direct measurement of the momentum transfer $t$ is possible. In comparison with the LRG data the FPS/LPS measurements have the advantages of negligible background from $p$ dissociation and a larger range in $x_{I P}\left(M_{X}\right)$. The disadvantages are the small acceptance and therefore low statistics, and a different source of systematic errors, namely the uncertainty in the $p$-beam optics.

Four data sets from H1 and ZEUS, listed and named in table 1, are used in the combination. The statistically dominant data set is H1 FPS HERA II, corresponding to the significant increase of luminosity after the HERA upgrade in the years 2001-2002. The ZEUS LPS detectors were not operated in the HERA II period. The bulk of the data $[5,6,2]$ was taken at electron and proton beam energies of $E_{e} \simeq 27.5 \mathrm{GeV}$ and $E_{p}=920 \mathrm{GeV}$, respectively, corresponding to an ep CM 
energy of $\sqrt{s}=318 \mathrm{GeV}$. The earlier ZEUS LPS 1 data, collected at $E_{p}=820 \mathrm{GeV}$, are corrected to the common $\sqrt{s}=318 \mathrm{GeV}$ by use of an extrapolation procedure.

The four data sets also show differences in the respective ranges of the kinematic variables, as seen in table 1 . In the individual analyses $[5,6,7,2]$ the reduced cross sections $\sigma_{r}^{D(3)}$ are directly measured for $t$-ranges visible to the proton spectrometers and extrapolated to the range ${ }^{1}$ $\left|t_{\text {min }}\right|<|t|<1 \mathrm{GeV}^{2}$, assuming an exponential $t$ dependence of the diffractive cross section and using the exponential slopes measured from the data. However, uncertainties of these experimental slope parameters were observed to lead to non-negligible tensions between the data sets. For the final combination a restricted common $t$-range is therefore defined by the LPS 2 visible $t$-range, $0.09<|t|<0.55 \mathrm{GeV}^{2}$, common to the bulk of both spectrometer data.

The original binning schemes of the $\sigma_{r}^{D(3)}$ measurements are very different. In the $\mathrm{H} 1$ case the measurements are extracted at fixed $\beta$, whereas for ZEUS the cross section is measured at fixed $M_{X}$; also the $Q^{2}$ and $x_{P}$ central values differ. Therefore, prior to the combination, the H1 and ZEUS data are transformed to a common grid of $\left(\beta, Q^{2}, x_{P}\right)$ points. The grid points are based on the original binning scheme of the high statistics FPS HERA II data. The $\left(Q^{2}, x_{I P}\right)$ grid points at the lowest $Q^{2}$ value of $2.5 \mathrm{GeV}^{2}$ and at the lowest and highest $x_{P}$ values, which are beyond the FPS HERA II data grid, are taken from the LPS 2 measurement.

Prior to the combination, the grid point measurements of the other data sets were swum to the common grid points using the dependences obtained from the respective NLO DPDF fits. Most of the corrections are smaller than $10 \%$, while a few points undergo corrections up to $\sim 30 \%$.

The correction factors from the visible $t$ range of the FPS HERA I and LPS 1 data samples to the restricted $t$ range are evaluated by using the $t$ dependences as a function of $x_{P}$ measured for each sample. The correction factors for the most precise FPS HERA II data are applied in bins of $\beta, Q^{2}$ and $x_{I P}$. For the LPS 2 sample the restricted range coincides with the visible range. Because of the uncertainties on the exponential slope parameters, such factors introduce uncertainties of $2.2 \%$, $1.1 \%$ and $5 \%$ for the respective FPS HERA II, FPS HERA I and LPS 1 data; these uncertainties are included in the normalisation uncertainty for each sample.

Figure 2a) shows the reduced cross sections from the four H1 and ZEUS data sets, after the corrections to $0.09<|t|<0.55 \mathrm{GeV}^{2}$ and after transformation to the common grid. A number to note is the ratio of the 'FPS HERA II' to the 'LPS 2' data averaged over the measured data points, which in the restricted $t$ range is $0.91 \pm 0.01$ (stat) \pm 0.03 (sys) \pm 0.08 (norm). Within the uncertainties, the ratio does not show any significant $\beta, Q^{2}$ or $x_{P}$ dependence.

\section{Data Combination}

To combine the separate $\mathrm{H} 1$ and ZEUS measurements the $\chi^{2}$ minimisation method developed in [8] was used. The simple basic (and natural) assumption is that at any given point in phase space the two experiments are measuring the same physics cross section. An important aspect of the method is the treatment of systematic errors. These are separated into correlated and uncorrelated errors, and the latter are in the minimisation included in the experimental errors, together with the statistical errors. The correlated systematic errors in contrast are fitted and thus serve to crosscalibrate the measurements. The resulting errors on the combined measurements are substantially

\footnotetext{
${ }^{1}$ The smallest kinematically accessible value of $|t|$ is denoted as $\left|t_{\text {min }}\right|$.
} 
H1 and ZEUS
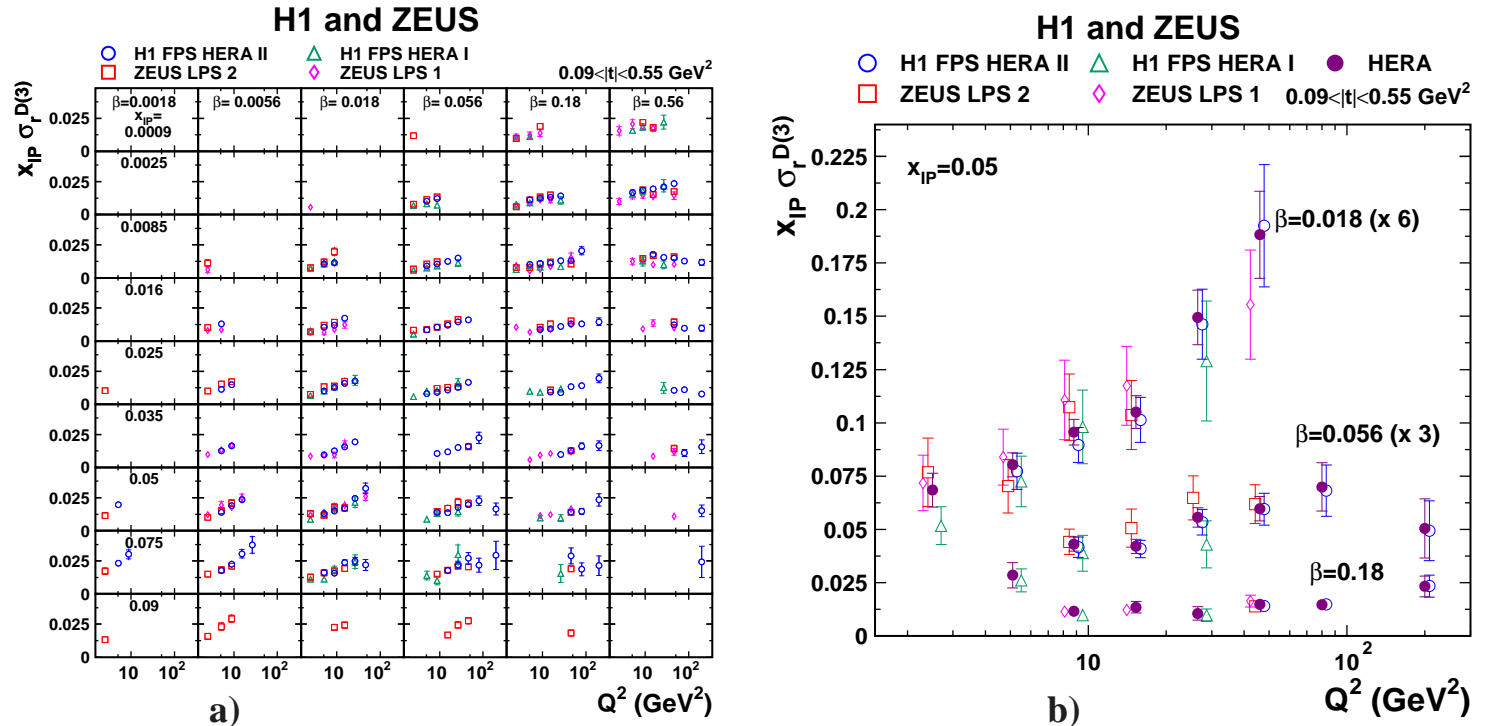

Figure 2: Reduced diffractive cross section $x_{\mathbb{P}} \sigma_{r}^{D(3)}\left(\beta, Q^{2}, x_{\mathbb{P}}\right)$ for $0.09<|t|<0.55 \mathrm{GeV}^{2}$ as a function of $Q^{2}$ for different values of $\beta$ and $x_{\mathbb{P}}$. In a) the H1 'FPS HERA II' [6], H1 'FPS HERA I' [5], ZEUS 'LPS 2' [2] and ZEUS 'LPS 1' [7] data are presented. The inner error bars indicate the statistical uncertainties, the outer error bars show the statistical and systematic uncertainties added in quadrature. Normalisation uncertainties are not included in the error bars of the individual measurements. In b) the combined data are compared to the $\mathrm{H} 1$ and ZEUS input data in 3 bins of $\beta$, at $x_{\mathbb{P}}=0.05$. Here the normalisation uncertainties are included in the error bars of the combined data points.

smaller, and moreover, the $\chi^{2} / n$.d.f. is a measure of the data consistency. The method is also model independent. This method was successfully used and extended in previous combinations of HERA measurements $[9,10]$. Note that due to the treatment of the correlated systematic errors, also those data points, which are unique to one sample, are affected and may be shifted in value and obtain better precision.

The $\chi^{2}$ is expressed as follows for the individual data sets:

$$
\chi_{\text {exp }}^{2}(\boldsymbol{m}, \boldsymbol{b})=\sum_{i} \frac{\left[m^{i}-\sum_{j} \gamma_{j}^{i} m^{i} b_{j}-\mu^{i}\right]^{2}}{\delta_{i, \text { stat }}^{2} \mu^{i}\left(m^{i}-\sum_{j} \gamma_{j}^{i} m^{i} b_{j}\right)+\left(\delta_{i, \text { uncor }} m^{i}\right)^{2}}+\sum_{j} b_{j}^{2}
$$

Here $\mu^{i}$ is the measured cross section value at the point $i\left(\beta_{i}, Q_{i}^{2}, x_{P, i}\right)$, and $\gamma_{j}^{i}, \delta_{i, \text { stat }}$ and $\delta_{i, \text { uncor }}$ are the relative correlated systematic, relative statistical and relative uncorrelated systematic uncertainties, respectively. The vector $\boldsymbol{m}$ of quantities $m^{i}$ expresses the values of the combined cross section for each point $i$ and the vector $\boldsymbol{b}$ of quantities $b_{j}$ expresses the shifts of the correlated systematic uncertainty sources, $j$, in units of the standard deviation. The relative uncertainties $\gamma_{j}^{i}$ and $\delta_{i, \text { uncor }}$ are multiplied by the combined cross section $m^{i}$ in order to take into account the fact that the correlated and uncorrelated systematic uncertainties are to a good approximation proportional to the central values (multiplicative uncertainties). On the other hand, the statistical uncertainties scale with the square root of the expected number of events, which is determined by the expected cross section, corrected for the biases due to the correlated systematic uncertainties. This is taken into account by the $\delta_{i, s t a t}^{2} \mu^{i}\left(m^{i}-\sum_{j} \gamma_{j}^{i} m^{i} b_{j}\right)$ term. 

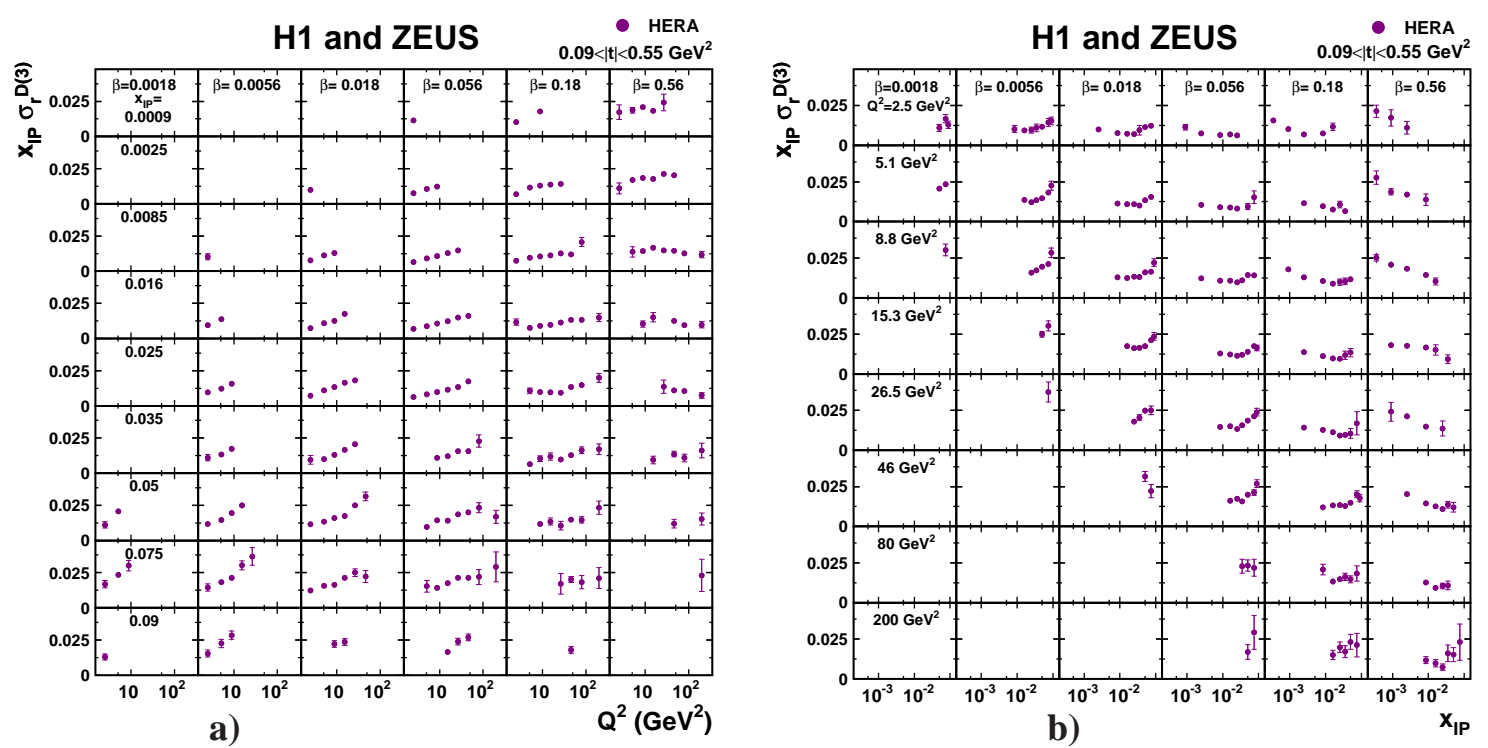

Figure 3: HERA combined reduced diffractive cross section $x_{\mathbb{P}} \sigma_{r}^{D(3)}\left(\beta, Q^{2}, x_{\mathbb{P}}\right)$ for $0.09<|t|<0.55 \mathrm{GeV}^{2}$. as a) a function of $Q^{2}$ for different values of $\beta$ and $x_{\mathbb{P}}$ and $\mathbf{b}$ ) as a function of $x_{\mathbb{P}}$ for different values of $\beta$ and $Q^{2}$. The error bars indicate the statistical, systematic and procedural uncertainties added in quadrature. The normalisation uncertainty is included.

If several analyses provide measurements at the same $\left(\beta, Q^{2}, x_{I P}\right)$ values, a $\chi_{\text {tot }}^{2}$ is built [10] from the sum of the $\chi_{\exp }^{2}$ of each data set, assuming the individual data sets to be statistically uncorrelated. The $\chi_{\text {tot }}^{2}$ is minimised with respect to the $m^{i}$ and $b_{j}$ from each data set with an iterative procedure.

\section{Results}

The result of the minimisation procedure is 191 cross section measurements, from 352 input data points. The data consistency is very good, with $\chi_{\min }^{2} / n_{\text {dof }}=133 / 161$. Most of the 23 correlated systematic uncertainties shift by less than $0.5 \sigma$ of the nominal value in the averaging procedure and with the exception of one none of them shifts by substantially more than $1 \sigma$.

The influence of several correlated systematic uncertainties is reduced significantly for the combined result. Notably, the uncertainty on the FPS proton energy measurement and the normalisation uncertainties on the 'FPS HERA I' and 'LPS 1' samples are reduced by more than a factor of 2. It is worthwhile to remark that since H1 and ZEUS use different reconstruction methods, similar systematic sources influence the measured cross section differently. Therefore, requiring the physics cross sections to be consistent at all $\left(\beta, Q^{2}, x_{P}\right)$ points constrains the systematic uncertainties efficiently. This cross-calibration effect leads to an average improvement of the experimental uncertainty of about $27 \%$ with respect to the most precise single data set, H1 FPS HERA II, though the latter contains five times more events than the second largest data set, ZEUS LPS 2. The correlated part of the experimental uncertainty is reduced from about 69\% in [6] to $49 \%$ in the combined measurement. The statistical, experimental and procedural uncertainties on the combined data are on average $11 \%, 13.8 \%$ and $2.9 \%$, respectively. The total uncertainty on the cross section is $14.3 \%$ on average and is $6 \%$ for the most precise points. The normalisation uncertainty, which contributes to the correlated systematic uncertainty on the combined data, is on average $4 \%$. 
The combined result extends the kinematic coverage with respect to the H1 and ZEUS measurements taken separately and the resulting cross section covers the region $2.5 \leq Q^{2} \leq 200 \mathrm{GeV}^{2}$, $0.0018 \leq \beta \leq 0.816$ and $0.00035 \leq x_{\mathbb{P}} \leq 0.09$, for $0.09<|t|<0.55 \mathrm{GeV}^{2}$. Figure $2 \mathrm{~b}$ ) shows the combined cross section as a function of $Q^{2}$ at $x_{I P}=0.05$, for three different values of $\beta$, compared with the individual measurements used for the combination. The reduction of the total uncertainty of the HERA measurement compared to the input cross sections is visible. Figures $3 a$ ) and $3 b$ ) show the HERA combined diffractive reduced cross sections as a function of $Q^{2}$ and $x_{T P}$, respectively. The derivative of the reduced cross section as a function of $\log \left(Q^{2}\right)$ decreases with $\beta$, a feature characteristic of the scaling violations in diffractive DIS, which are now measured precisely from proton-tagged as well as LRG data.

\section{Conclusions}

The reduced diffractive cross sections, $\sigma_{r}^{D(3)}(e p \rightarrow e X p)$, measured by the H1 and ZEUS collaborations by using proton spectrometers to detect the leading protons, are combined. The four input data sets from the two experiments are consistent with a $\chi_{\min }^{2} / n_{\text {dof }}=133 / 161$. The combination of the measurements results in more precise and kinematically extended diffractive DIS data in the $t$-range $0.09<|t|<0.55 \mathrm{GeV}^{2}$. The total uncertainty on the cross section measurement is $6 \%$ for the most precise points. The combined data provide the to date most precise determination of the absolute normalisation of the $e p \rightarrow e X p$ cross section.

\section{Acknowledgement}

Many thanks to all colleagues in H1 and ZEUS for providing the material in this report and for help given in its preparation. Warm thanks also to the Marseille team for the excellent organisation and the pleasant atmosphere of this conference.

\section{References}

[1] G. Ingelman and P.E. Schlein, Phys. Rev. Lett. 152B (1985) 256; A. Donnachie and P.V. Landshoff, Nucl. Phys. B303 (1988) 634.

[2] ZEUS Collaboration, S. Chekanov et al., Nucl. Phys. B816, 1 (2009).

[3] H1 Collaboration, A. Aktas et al., Eur. Phys. J. C48, 715 (2006).

[4] H1 Collaboration, A. Aktas et al., Eur. Phys. J. C72, 2175 (2012).

[5] H1 Collaboration, A. Aktas et al., Eur. Phys. J. C48, 749 (2006).

[6] H1 Collaboration, A. Aktas et al., Eur. Phys. J. C71, 1578 (2011).

[7] ZEUS Collaboration, S. Chekanov et al., Eur. Phys. J. C38, 43 (2004).

[8] A. Glazov, AIP Conf. Proc. 792, 237 (2005); Talk at DIS2005, Madison, Wis., USA, 27.4 - 1.5.2005

[9] H1 and ZEUS Collaborations, F. D. Aaron et al., JHEP 1001, 109 (2010).

[10] H1 Collaboration, F. D. Aaron et al., Eur. Phys. J. C63, 625 (2009). 